\title{
LA SITUACIÓN RESIDENCIAL DE LA POBLACIÓN INMIGRANTE EN ANDALUCÍA
}

\author{
SEBASTIAN RINKEN y ANAÏS HERRÓN \\ Instituto de Estudios Sociales de Andalucía (IESA-CSIC). Córdoba
}

PALABRAS CLAVE ADICIONALES

Vivienda, exclusión espacial, inmigración, encuesta.

\section{ADDITIONAL KEYWORDS}

Housing conditions, Spatial exclusion, Immigration, Survey.

RESUMEN: En este artículo se describe la situación residencial en Andalucía de los inmigrantes procedentes de países menos desarrollados. Basándonos en datos de una reciente encuesta representativa, llevamos a cabo un examen pormenorizado de las situaciones de ausencia de vivienda digna en esta población, haciendo especial hincapié en establecer definiciones claras e indicadores transparentes. Los resultados confirman la existencia de una situación residencial gravemente deficiente de una proporción importante de los inmigrantes asentados en zonas de agricultura intensiva, sobre todo en la provincia de Almería. Sin embargo, a diferencia de otros estudios, concluimos que aproximadamente cuatro de cada cinco inmigrantes asentados en Andalucía disponen de una vivienda digna.

ABSTRACT: In recent years, a variety of observers have denounced the housing conditions of Andalusia's growing immigrant population as largely miserable. In addition to the overcrowding in and the poor quality of housing available to immigrants, special emphasis has been placed on the confinement to extra-urban spaces in Andalusia's thriving areas of intensive agriculture, specifically in Almeria province. Based on survey data, this article provides a far more reliable measurement of these three phenomena (overcrowding, poor quality housing and spacial exclusion) than previously available, including social profiles of the people affected by these deficiencies. Our results point to the existence of sizable and serious problems with housing conditions specifically in this population. However, differently from previous studies, our data show that these particular problems affect a minority only of Andalusia's immigrants.

E-mail: srinken@iesaa.csic.es

\section{Revista Internacional de Sociología (RIS)}

Tercera Época, No 38, Mayo-Agosto, 2004, pp. 101-125. 
RIS

REVISTA INTERNACIONAL DE SOCIOLOCIA

№ 38, MAYO-ACOST0, 2004

SEBASTIAN RINKEN Y ANAÏS HERRÓN

\section{INTRODUCCIÓN}

El acceso a la vivienda digna es, junto a la alimentación, una de las necesidades básicas del ser humano. La ausencia de un domicilio que reúna condiciones mínimas de espacio y salubridad significa estar expuesto a situaciones como las inclemencias meteorológicas, la falta de intimidad personal y familiar, la imposibilidad de descanso imperturbado y la falta de condiciones higiénicas adecuadas. La Declaración Universal de los Derechos Humanos (artículo 25, apd. 1) y las Constituciones de muchos países democráticos, entre ellas la Constitución espanola (artículo 47), definen el acceso a la vivienda como un derecho.

El alcance práctico de este derecho depende de la configuración de las políticas públicas de la vivienda en cada país, cuya función consiste, por un lado, en evitar una desvinculación excesiva del precio de la vivienda libre respêcto a los ingresos, y por otro, en contar con mecanismos específicos que permitan disponer de una vivienda digna también a los segmentos económicamente más desfavorecidos de la población (por ejemplo, mediante el alquiler, con precios reducidos, de viviendas de protección oficial) (Ponce, 2004). En términos comparativos, la situación actual en España destaca por una serie de factores que complican el acceso a la vivienda, sobre todo para personas que no tienen ingresos estables (Sánchez Hernández, 2002): el bajo porcentaje de viviendas en alquiler (que no llega al $15 \%$ del parque de viviendas), el vertiginoso crecimiento de los precios de la vivienda libre en los últimos años y el papel residual de la vivienda de protección oficial. Asimismo, visto que "la movilidad residencial decrece con la renta y con la clase social" (Leal, 2002: 67), la coyuntura actual del mercado inmobiliario español acentúa la distribución espacial de la población en función de su poder adquisitivo, posiblemente fomentando procesos de concentración de personas expuestas al riesgo de exclusión social (Pérez Yruela, Sáez Méndez y Trujillo Carmona, 2002).

En relación con la reciente conversión de España en país de inmigración (Izquierdo, 1996; Arango, 2000), cabe predecir, pues, que el acceso a la vivienda constituya uno de los principales problemas de los inmigrantes económicos, ya que si el acceso a la vivienda es difícil para amplios segmentos de la población autóctona, lo más probable es que la población inmigrante tenga esas mismas o mayores dificultades, sobre todo en la primera fase de su estancia. Entre las posibles dificultades añadidas, cabe mencionar la ausencia de un estatus administrativo regularizado, la obtención de un trabajo normalizado y bien remunerado (Hoggart y Mendoza, 1999; Abad Márquez, 2002; Cachón, 2003; Gualda Caballero, 2003; Izquierdo, 2003), la carencia de una red social de apoyo y un escaso acceso a las políticas públicas sectoriales.

Ahora bien, la literatura disponible sobre la situación residencial de los inmigrantes en España suele enfocar el fenómeno objeto de estudio desde otra perspectiva, a saber: el conflicto entre las poblaciones autóctona e inmigrante cuyo 
caso emblemático es el estallido de El Ejido (Almería) de febrero de $2000^{1}$. En este contexto, la situación residencial de la cada vez más numerosa población inmigrante en Andalucía ${ }^{2}$ se ha convertido en objeto de una serie de informes y publicaciones cuya tónica suele ser dramática. Sin entrar en las diferencias de enfoque, método y rigor, de esta literatura emerge una descripción común:

(a) Los inmigrantes viven mayoritariamente ${ }^{3}$ en infraviviendas localizadas en diseminado, en muchos casos estando además hacinados en las mismas; la minoría que ha logrado asentarse en núcleos urbanos suele hacerlo en situación de hacinamiento y, en muchos casos, en inmuebles deteriorados con graves deficiencias de equipamiento ${ }^{4}$.

(b) En la provincia de Almería, debido bien a la "negativa de los habitẳntes autóctonos a alquilarles una vivienda en el núcleo urbano" a los inmigrantes (SOS Racismo, 2001: 27), bien a la delimitación excluyente de los espacios en las ciudades (Martín Díaz et al., 1999: 142), "los espacios de copresencia casi han desaparecido" (Checa Olmos y Arjona Garrido, 2002: 665), de manera que está emergiendo una situación de segregación espacial.

Constatamos una serie de deficiencias metodológicas que aconsejarían cierta prudencia a la hora de aceptar esta visión establecida como un reflejo válido de la realidad. Concretamente, destacaríamos los siguientes problemas:

\footnotetext{
' Para una crónica e interpretación de los sucesos desde una óptica antirracista, véase SOS Racismo (2001); para una visión enfática con la óptica de los agricultores autóctonos, véase Azurmendi (2001); para un análisis de los discursos surgidos para explicar el conflicto, véase Terrén (2003). Entre las primeras publicaciones sobre la situación residencial de los inmigrantes en España, destacan MTAS (1996) y Martínez Veiga (1999).

${ }^{2}$ A partir de un nivel inicial bajo, la población inmigrante procedente de países menos desarrollados ha crecido rápidamente en los últimos años, concentrándose esencialmente en el litoral mediterráneo (provincias de Málaga y Almería) y triplicando su volumen entre 2000 y 2003 (Rinken et al., 2003).

${ }^{3}$ Según Checa Olmos (2002: 105), el 65\% de los inmigrantes extracomunitarios en Almería viven en diseminado, mientras que Castaño Madroñal (2000: 65; 2002: 119) cifra en el 80\% la proporción de la población inmigrante del Poniente almeriense viendo "en disperso", "en cortijos y almacenes diseminados". Según Checa Olmos y Arjona Garrido (2002: 659) hasta el 80\% de los temporeros inmigrantes en Huelva están residiendo en diseminado.

${ }^{4}$ Según el Defensor del Pueblo Andaluz, en Almería, "entre el 60\% y el 80\%" de las viviendas y alojamientos destinados a trabajadores inmigrantes tienen la consideración de infraviviendas (DPA, 2001: 101). En cuanto a la ocupación de estas viviendas o alojamientos, "lo normal es que la vivienda sea compartida por un elevado número de trabajadores" (Gordo Márquez, 2002: 104, en referencia a la provincia de Huelva). Varios observadores señalan índices de hacinamiento "a razón de 2 ó 3 personas por dormitorio" (DPA, 2001: 24; Checa Olmos, 2002: 110), y en algunos casos se describen incluso “cortijos que 'albergan' hasta 40 personas" (Martín Díaz et al., 1999: 141).
} 
RIS

REVISTA INTERNACIONAL DE SOCIOLOCIA

No 38, MAYO-AGOSTO, 2004

SEBASTIAN RINKEN y ANAÏS HERRÓN

- Opacidad e insuficiencia de procedimientos. Algunos estudios se nutren - directa o indirectamente, a través de trabajo de campo y entrevistas a informantes cualificados - de un profundo conocimiento de un territorio concreto. No obstante, en cuanto a la cuantificación de los fenómenos descritos, la - generalmente, escasa - información disponible acerca de los procedimientos no permite clasificar éstos como medición, visto que suele tratarse de observaciones que carecen bien de fuente contrastable, bien de representatividad.

- Generalizaciones injustificadas. Es habitual la generalización sucesiva - "sin justificación aparente", como apunta Pumares (2001: 359) — de unidades de observación identificables - por ejemplo: los magrebíes asentados en el municipio de El Ejido- a ámbitos territoriales y conjuntos de población siempre más amplios, de manera que, de forma explicita o implícita, lăs carencias observadas llegan a atribuirse a toda la población inmigrante asentada en Andalucía.

- Falta de definiciones. Se detecta también una ausencia llamativa de definiciones. Términos clave como "segregación" se manejan sin explicar su significado exacto. La tendencia general es utilizar "segregación espacial" como sinónimo de ubicaciones en diseminado, sin atribuir mayor relevancia a matizaciones emergentes en relación con este último término. Así, en vez de sólo mencionarla (DPA, 2001: 22), a efectos de medición e interpretación sería a todas luces crucial profundizar en la diferencia entre tipologías edificatorias sin conexión con el casco urbano principal, por un lado, y pequeños núcleos de población y sus periferias, por otro. Asimismo, no cabe descartar la posibilidad de que la distancia del casco o núcleo urbano constituya un elemento clasificatorio importante; no resulta satisfactorio simplemente mencionar (DPA, 2001: 44) la preferencia de los inmigrantes por "no residir en lugares alejados del núcleo urbano, como máximo a una distancia, aproximada, de 3 kilómetros ". A falta de matices, la literatura disponible tiende a equiparar la concentración de los inmigrantes en espacios distintos de los habitados por la población autóctona, por un lado, y el alejamiento de la población inmigrante de los núcleos urbanos, por otro.

Proponemos limitar el significado del término "segregación espacial" a la separación y concentración residencial de las poblaciones en función de su procedencia. Asimismo, sugerimos utilizar el término de "exclusión espacial" para indicar el alejamiento de los núcleos urbanos y la consecuente falta de acceso a determinados servicios básicos. Finalmente, sugerimos definir como "vivienda digna" cualquier situación residencial que no tenga carencias graves en cuanto a: el tipo de vivienda y su equipamiento (infravivienda), el ratio de ocupación (hacinamiento), o el acceso a las infraestructuras básicas 
(exclusión espacial). Nuestro objetivo en este artículo es describir de manera sistemática la situación residencial de la población inmigrante en Andalucía, centrándonos en la falta de vivienda digna ${ }^{5}$.

\section{METODOLOGÍA}

Este artículo se basa en los resultados de una encuesta sobre las características y necesidades de la población inmigrante asentada en Andalucía, realizada en la primavera de 2003 en el marco del proyecto de investigación "Necesidades de la Población Inmigrante en Andalucía" (NEPIA) ${ }^{6}$. Es menester señalar que excluimos de la población objeto de estudio a personas nacidas en el espacio económico de la Unión Europea u otros países con un nivel de desarrollo notoriamente elevado (EE.UU., Canadá, Australia, Japón, entre otros), obviando así la inmigración "de países ricos" que existe en algunas zonas de Andalucía (sobre todo, la Costa

\footnotetext{
${ }^{5}$ En este artículo, la diferencia entre "vivienda" y "alojamiento" no tendrá mayores implicaciones operativas, al medirse la calidad de la situación residencial con un solo conjunto de indicadores. Cabe mencionar que nos constan dos maneras distintas de desmarcar "alojamiento" de "vivienda": (a) en relación con la duración o estabilidad de la permanencia, de naturaleza transitoria en el caso del alojamiento, y (b) en relación con la tipología habitacional en cuestión, tipología que, en el caso del alojamiento y si no se trata de infravivienda manifiesta, se encuentra a medio camino entre vivienda y residencia colectiva.Vease Cortés Alcalá (1997) para un concepto más amplio de "exclusión residencial".

${ }^{6}$ El proyecto NEPIA fue realizado entre los años 2002 y 2003 por el Instituto de Estudios Sociales de Andalucía (IESA) por encargo de la Consejería de Gobernación de la Junta de Andalucía (Dirección General de Coordinación de Políticas Migratorias) con financiación del Fondo Social Europeo. Entre las muchas personas que colaboraron en las actividades de investigación a raíz de las que se pudo redactar este artículo, cabe destacar, en primer lugar, al Investigador Principal de NEPIA, Manuel Pérez Yruela, a quien agradecemos también sus observaciones sobre una anterior versión de este artículo. En segundo lugar, agradecemos a los demás miembros del equipo central de NEPIA (Elisa Rodriguez Ortiz, Thierry Desrues y Luis Rodriguez Morcillo-Baena) y la Unidad Técnica del IESA (especialmente a Mari Carmen Abril, Paco Aranda, Begoña Buiza, Carmela Gutiérrez, Rafaela Sotomayor y Manuel Trujillo) su papel esencial en la realización de la encuesta. En tercer lugar, es necesario reconocer la labor de los coordinadores provinciales del proyecto (Almería: Matilde Asensio García y Juan Sebastián Prados; Cádiz: Cristina Marcos Montiel; Córdoba y Jaén: Raquel Martínez Chicón; Granada: Javier Rosón Lorente; Huelva: Esther Márquez Lepe; Málaga: Lourdes Alba Fernández; Sevilla: Rocío Medina Martín) y del Comité Asesor del Proyecto NEPIA (compuesto por Teresa Bravo Dueñas, Francisco Checa Olmos, Javier García Castaño, Bernabé López García, Isidro Maya Jariego, Alberto Morillas Fernández y Josefina Pereira Lorenzo). Quisiéramos agradecer especialmente la colaboración de todas las personas entrevistadas. A la Dirección General de Coordinación de Políticas Migratorias de la Junta de Andalucía, agradecemos la confianza puesta en el IESA al encargarnos la realización de NEPIA. Reconocer finalmente la cofinanciación aportada al mismo por el Ministerio de Ciencia y Tecnología español, siendo el coordinador general de NEPIA, Sebastian Rinken (y coautor de este artículo) beneficiario del programa Ramón y Cajal.
} 
del Sol). Por tanto, en este artículo (y en todo el estudio NEPIA), el término "inmigrante" no equivale a "extranjero", sino a "inmigrante procedente de países menos desarrollados" 7 . Una vez constatada la ausencia de fuentes fiables que cuantifiquen el universo de referencia así definido, decidimos utilizar datos del Padrón Municipal Continuo como aproximación a dicho universo poblacional; concretamente, combinamos datos del padrón de 1 de enero del 2000 (último padrón publicado por el INE en aquel momento) y del Padrón Continuo de 150 municipios andaluces con presencia significativa, previamente seleccionados a partir de las cifras del 2000, municipios de los que recogimos datos actualizados a otoño de 2002. Para aprovechar al máximo el tamaño total de la muestra (1800 entrevistas), apostamos por una estratificación cruzada según zona geopolítica de procedencia (cinco categorías) y zona socioeconómica de asentamiento (cuatro categorías), obteniendo así la posibilidad de comparar, con una representâtividad estadística satisfactoria ${ }^{8}$, los datos de estas subcategorías, dotadas con cuota fija independientemente de su peso en el Padrón Municipal. Asimismo, se aplicaron cuotas proporcionales por sexo'.

Los cinco conjuntos geopolíticos de procedencia quedaron definidos de la siguiente manera: (a) Magreb y Oriente Próximo; (b) África subsahariana; (c) Latinoamérica; (d) Países del Este de Europa (no UE / EEE); y (e) Asia (excepto Oriente Próximo y Japón). Para dar una orientación sobre el peso que tienen estos conjuntos geopolíticos de procedencia en la población inmigrante procedente de países menos desarrollados, cabe decir que, en Andalucía, los conjuntos de Magreb (fundamentalmente, marroquíes) y de Latinoamérica (sobre todo argentinos, ecuatorianos y colombianos) agrupan cada uno a aproximadamente un tercio de dicha población, mientras que el último tercio se reparte entre países de origen agrupados en los restantes tres conjuntos geopolíticos.

Las cuatro categorías de zonas socioeconómicas de asentamiento son las siguientes: (a) Grandes ciudades (capitales de provincia y cinturón metropolitano); (b) Zonas costeras con economía dominada por el turismo; (c) Zonas costeras con economía dominada por la agricultura intensiva; y (d) Zonas rurales del interior (esencialmente dedicadas a la agricultura extensiva). Estas categorías se utilizaron para clasificar todos los municipios andaluces en cuanto a la realidad socioeconómica dominante, considerando que ésta define en gran medida el perfil

\footnotetext{
${ }^{7}$ Nótese que, en Andalucía, la segunda generación de inmigrantes (nacida en España) es aún incipiente y generalmente limitada a edades inferiores a la edad mínima para participar en la encuesta (16 años).

${ }^{8}$ En el supuesto de muestreo aleatorio, no aplicable con propiedad en este estudio, el nivel de error para un nivel de confianza del $95 \%$ sería de $+/-5,2 \%$ para las subcategorías de procedencia y alrededor del $3 \%$ para toda la muestra en su conjunto.

${ }^{9}$ Para la ponderación de la muestra, la Unidad Técnica del IESA estableció coeficientes mediante calibración con el programa WesVar 4.2.
} 
de oportunidades, problemas y necesidades con el que se enfrentan típicamente los inmigrantes ${ }^{10}$. De esta manera, estamos en condiciones de comparar entre dos categorías que reflejan realidades urbanas (costa turística y grandes ciudades), frente a otras dos que se refieren esencialmente al mundo rural (agricultura intensiva y rural interior). En cuanto a su referencia territorial, "costa turística" engloba prácticamente todo el litoral malagueño excepto la capital provincial, junto con algunos municipios de Granada y Almería, mientras que "agricultura intensiva" se refiere principalmente al litoral almeriense, ${ }^{11}$ junto con algunos municipios de Huelva y Granada. Para dar una idea del peso ponderado de estas cuatro categorías, las zonas de "agricultura intensiva" y "costa turística" agrupan cada una a aproximadamente un cuarto de la población inmigrante empadronada en Andalucía, mientras que en las "grandes ciudades" reside alrededor del $40 \%$; con un $13 \%$, el peso del "rural interior" es menor en esta población. La muestra definitiva se compone de 1797 personas entrevistadas (58,1\% hombres y $41,9 \%$ mujeres).

En cuanto a la recogida de datos, la elaboración de un instrumento estandardizado de medición apto para una muestra tan heterogénea en cuanto a su procedencia, características culturales y situación social, ha constituido un desafío metodológico considerable (Maya Jariego, 2001). En NEPIA hicimos un especial esfuerzo por diseñar un cuestionario que cumpliese con los criterios de amplitud temática, flexibilidad (apartados filtrados en función de la situación especifica del entrevistado), sencillez y comparabilidad, entre otros. La administración del cuestionario se encargó a equipos de campo creados y formados específicamente para este proyecto, en muchos casos compuestos por inmigrantes con amplios conocimientos lingüísticos y afinidad cultural con la población a entrevistar. Asimismo, diseñamos un exigente procedimiento de control de calidad, incluyendo llamadas telefónicas a un $40 \%$ de los entrevistados.

El cuestionario fue administrado de manera presencial por el equipo de campo a personas captadas de forma accidental, según las cuotas previstas, en un total de 108 municipios. Para evitar sesgos, diversificamos esta búsqueda de entrevistados definiendo cuatro ámbitos distintos de captación (laboral, residencial, recreativo y asociativo), con cuotas mínimas y máximas para los primeros tres de ellos, en relación con el total de entrevistas realizadas por cada entrevistador. De esta manera, aun sin haber obtenido una captación aleatoria propiamente dicho, consideramos que los datos de esta encuesta tienen una representatividad y validez muy razonables ${ }^{12}$.

\footnotetext{
${ }^{10}$ Agradecemos a Manuel Trujillo, de la Unidad Técnica del IESA, habernos facilitado esta clasificación de todos los municipios andaluces en relación con las cuatro categorías mencionadas, establecidas por él en función de datos censales sobre nivel de educación, actividades socioeconómicas y estado urbanístico, entre otras variables.

"El grueso del trabajo de campo relativo a esta categoría (zona de agricultura intensiva, provincia de Almería) se realizó en los municipios de Roquetas, El Ejido, Nijar, Vícar y La Mojonera.

${ }^{12}$ Para una descripción más detallada de la encuesta NEPIA, remitimos a Rinken (2003).
} 
R IS

REVIST'A INTERNACIONAL, DE SOCIOLOGIA

No 38, MAYO-AGOSTO, 2004

SEBASTIAN RINKEN Y ANAÏS HERRÓN

\section{RESULTADOS}

En este apartado se describe con cierto detalle la situación residencial de los inmigrantes procedentes de países menos desarrollados asentados en Andalucía. En primer lugar, examinaremos indicadores sobre tres situaciones concretas de vivienda "no digna", relativos a: (a) tipo y equipamiento del hogar, (b) ocupación (número de habitantes por unidad habitacional), y (c) equipamiento de la zona o barrio. Estos grupos de indicadores permitirán cuantificar tres tipos distintos de carencias, a saber: situaciones de infravivienda, hacinamiento y exclusión espacial, respectivamente. Señalaremos en cada caso la cuantificación, la distribución geográfica (según zonas socioeconómicas de asentamiento y ocasionalmente también según provincias) y el perfil social de los afectados. Además de las tres variables fundamentales para el muestreo (sexo, "zona geopolítica de procedencia" y "zona socioeconómica de asentamiento") nos referiremos también a la variable "año de llegada", que nos permitirá examinar el efecto de la duración del asentamiento en Andalucía en la situación residencial ${ }^{13}$, y la variable "ingresos personales", que hará posible una aproximación a la situación económica de los inmigrantes y su efecto para la situación residencial. Concluiremos este apartado de resultados examinando la posible acumulación de estas carencias y, finalmente, calibrando nuestros resultados en relación con otros estudios existentes.

En este artículo, nuestra herramienta fundamental será el contraste de proporciones entre distintas subcategorías de la muestra. Para la casi totalidad de estas comparaciones, los resultados son estadísticamente significativos; sólo en muy contadas ocasiones utilizamos datos que carecen de significación estadística por considerarlos particularmente interesantes. Cabe subrayar que "estadísticamente significativo" quiere decir que es improbable que sea casual la diferencia observada en una subcategoría de la muestra, respecto de la media; sin embargo, tal constatación no implica por si misma la confirmación de un vínculo causal.

\section{1) Las situaciones de infravivienda}

Hemos definido como persona en situación de infravivienda a cualquier entrevistado que declarase, en respuesta a las correspondientes preguntas de nuestra encuesta:

- no tener domicilio ${ }^{14}$;

- vivir en un tipo de alojamiento clasificado como "precario"; o

- no tener acceso, en su domicilio habitual, a uno o varios de los equipamientos más básicos.

\footnotetext{
${ }^{13}$ Cabe señalar que generalmente, según nuestros datos, la llegada a Andalucía coincide prácticamente - $y$ en medida siempre mayor conforme nos aproximemos a la actualidad — con la llegada a España.

${ }^{14}$ En el cuestionario NEPIA, el apartado dedicado a la vivienda empieza con la pregunta: “¿Cuánto
} 
Cualquiera de las tres condiciones es suficiente para clasificar la situación residencial de "infravivienda", ya que se trata de requisitos mínimos para una vivienda digna ${ }^{15}$. Por tanto, antes de cuantificar el fenómeno de la infravivienda, así definido, en su conjunto, dedicaremos breves notas a sus tres componentes.

\section{Extensión y localización del fenómeno}

Sin domicilio: La falta de alojamiento es el componente de la infravivienda que afecta al menor número de personas, ya que sólo alcanza al $0,5 \%$, exclusivamente hombres que residen en zonas de agricultura intensiva o extensiva y en las ciudades, pero en ningún caso en las zonas turísticas costeras.

Tipo de vivienda o de alojamiento: El tipo de vivienda que declararon tener los inmigrantes se ha agrupado en tres categorías, a saber: "vivienda completa", "habitación" o "precariedad", la última de las cuáles es considerada automáticamente como infravivienda. Alrededor de un $85 \%$ de los entrevistados reside en viviendas "completas", mayoritariamente en pisos (unifamiliares o compartidos) en alquiler, aunque cerca de un $15 \%$ declara vivir en casas o adosados. En cuanto a los que viven en una "habitación" (un 8,5\% del total de entrevistados), la mayoría de ellos tiene una habitación subarrendada, cerca de un tercio (sobre todo mujeres) dijo vivir en una habitación en casa de los patrones, y menos de un 10\% (casi todos varones) contestó estar alojado en un hotel, pensión u hostal. Nuestra clasificación prevé la categoría de "precariedad" manifiesta, con graves deficiencias de habitabilidad e infraestructura, como son los centros de transeúntes u otro tipo de residencia colectiva precaria; chabolas, casas abandonadas, naves industriales o similares; el alojamiento en un coche o tienda de campaña; y finalmente, vivir "sin techo" alguno ${ }^{16}$. El 5,7\% de los encuestados indicó vivir en situaciones manifiestamente precarias, entre las cuales las chabolas, casas abandonadas, naves industriales o similares son las más extendidas. Cabe destacar que el 13,5\% de los

\footnotetext{
tiempo lleva viviendo en su domicilio actual?". Los entrevistados que indicaron no tener domicilio no contestaron, lógicamente, las preguntas relativas a la descripción de la vivienda.

${ }^{15}$ En su artículo 62, el IV Plan Andaluz de Vivienda y Suelo (BOJA núm. 117/2003) define como infravivienda cualquier edificación que presente una de las siguientes carencias: "(a) No poseer instalaciones sanitarias básicas, abastecimiento de agua, saneamiento, electricidad o iluminación y ventilación suficientes; (b) Manifestar condiciones de la edificación por debajo de los requerimientos mínimos, en relación con los aspectos constructivos. (c) Presentar un alto grado de hacinamiento de los moradores de la vivienda." Hemos preferido tratar por separado el tercero de estos aspectos (hacinamiento); los primeros dos se corresponden — con ligeros matices- a nuestra definición de infravivienda.

${ }^{16}$ Opción aplicable, en esta pregunta, sólo a los poquísimos entrevistados sin domicilio que no habían señalado anteriormente dicha situación.
} 
RIS

REVISTA INTERNACIONAL DE SOCIOLOCIA

№ 38, MAYO-AGOST0, 2004

SEBASTIAN RINKEN y ANAIISS HERRÓN

entrevistados en la zona de agricultura intensiva indicó vivir en hogares obviamente precarios. Casi todos estos entrevistados son varones, de manera que el alojamiento en unidades habitacionales clasificables de entrada como "precarias" afecta aproximadamente a uno de cada cinco inmigrantes varones asentados en zonas de agricultura intensiva. Por otro lado, en las zonas costeras turísticas esta modalidad es prácticamente inexistente.

Equipamiento de la vivienda: Como tercer elemento de nuestra definición de infravivienda, creemos necesario hacer referencia a las posibles carencias de equipamientos básicos en una unidad residencial perteneciente, en principio, bien a la categoría de "vivienda completa", bien a la categoría de "habitación". Para ello utilizamos una pregunta diseñada para comprobar el acceso a una serie de equipamientos en estas dos categorías de hogares. De los once ítems incluidos en esta pregunta, hemos considerado esencial para la clasificación como "vivienda digna" los siguientes: (a) tener una instalación de agua caliente; (b) disponer de baño o ducha, y (c) disponer de instalación eléctrica. La ausencia de cualquiera de estos tres equipamientos ${ }^{17}$ nos indica que la vivienda no está suficientemente equipada, pasando por tanto a clasificarse como "infravivienda" 18 . Ahora bien, de entre los entrevistados residentes en una "vivienda" o "habitación", el porcentaje que carece de alguno de estos equipamientos básicos no es demasiado elevado, ya que sólo alcanza al 2,9\%. Entre ellos, la falta de agua caliente es la situación más extendida, puesto que prácticamente todos estos entrevistados tienen acceso a baño o ducha y disponen de instalación eléctrica. En las zonas de agricultura intensiva se registra el mayor número de carencias: un 6,4\% de los entrevistados con vivienda supuestamente "normalizada" declararon no tener alguno de los tres equipamientos que hemos considerado básicos. El agua caliente vuelve a ser la carencia más frecuente, afectando al 5,3\% de los entrevistados en esta zona, mientras que el $2,8 \%$ no tienen baño o ducha.

Extensión y localización de la infravivienda. Como suma de las tres subcategorías de infravivienda que acabamos de comentar, resulta que la situación de infravi-

\footnotetext{
${ }^{17}$ Nuestros indicadores cubren cuatro de los cinco ítems incluidos por el Defensor del Pueblo Andaluz en las fichas de visita a las viviendas de inmigrantes manejadas, en el año 2000, durante el trabajo de campo para conocer su estado de habitabilidad (DPA, 2001: 129), puesto que la disponibilidad de "agua caliente" presupone la de agua corriente. Con este mismo matiz, nuestros indicadores coinciden también con la lista de los equipamientos más básicos utilizadas por la Fundación FOESSA (1998: 291).

${ }^{18} \mathrm{Hemos}$ prescindido de incluir indicadores acerca del estado general del edificio (humedades, grietas, etc.), al considerar que pueden inducir a valoraciones muy subjetivas y clasificaciones poco fiables.
} 
vienda afecta al $8,9 \%$ de la muestra ponderada. A tenor de los datos referidos hasta ahora, no sorprenderá que sean las zonas de agricultura intensiva donde se observa dicho fenómeno en mayor medida. Según nuestros datos, uno de cada cinco (el 20,1\%) de los inmigrantes asentados en estas zonas habita en lo que hemos denominado, según el procedimiento descrito, una "infravivienda". Dentro de esta categoría de "zona de agricultura intensiva", los correspondientes municipios de la provincia de Almería son los más afectados por la infravivienda, ya que, en ellos, se alcanza un porcentaje incluso superior (el $22,2 \%$ de los entrevistados) ${ }^{19}$. Por otro lado, en las zonas turísticas costeras, el porcentaje de inmigrantes que viven en situación de infravivienda es prácticamente residual, afectando sólo al $1,1 \%$ de ellos ${ }^{20}$.

\section{Infravivienda: perfil social de los afectados}

La infravivienda es un fenómeno que afecta principalmente a los varones. Observando las diferencias en el conjunto de Andalucía, el 13\% de los inmigrantes varones reside en una infravivienda, frente al 3,6\% de mujeres. Estas diferencias se acentúan en algunas zonas socioeconómicas de asentamiento, sobre todo en la de agricultura intensiva, donde la proporción de los inmigrantes varones residentes en infravivienda se acerca al $30 \%$ (frente a un 10\% aproximadamente de las mujeres). Asimismo, se observan importantes diferencias por sexo en algunos conjuntos geopolíticos, destacando el conjunto de "Magreb y Oriente Próximo", con el $22 \%$ de varones y el $7 \%$ de mujeres, respectivamente, afectados por situaciones de infravivienda ${ }^{21}$.

Las situaciones de infravivienda se concentran, precisamente, en los inmigrantes procedentes del Magreb y Oriente Próximo y del África Subsahariana, con un $17 \%$ y un $13 \%$, respectivamente, de afectados. Si estos dos colectivos contrastan con la media global por su alto porcentaje, los iberoamericanos y los asiáticos lo hacen en sentido contrario: los primeros están afectados en un 1,8\% por la infravivienda, y el porcentaje de asiáticos es aún menor, pues sólo alcanza el $0,2 \%$.

${ }^{19}$ A pesar de no ser significativo estadísticamente, debido al número de entrevistas realizadas, mencionamos, como contraste interesante, que en la provincia de Huelva, en el mismo tipo de municipios, sólo se ven afectados por la infravivienda un 12,5\% de los entrevistados.

${ }^{20}$ Estando ubicados todos estos casos en la provincia de Granada, es posible que se trate de municipios que, a pesar de haberse clasificado como pertenecientes a la "costa turistica", participan también en el despegue de la agricultura intensiva bajo plástico que se está observando recientemente en el litoral de esta provincia.

${ }^{21}$ En el resto de zonas y conjuntos las diferencias entre varones y mujeres son menores y en algunos casos no son estadísticamente significativas. 
RIS

REVISTA INTERNACIONAL DE SOCIOLOCIA

№ 38, MAYO-AGOSTO, 2004

SEBASTIAN RINKEN y ANAIIS HERRÓN

Según el año de llegada a Andalucía, resulta que los inmigrantes que llevan más de ocho años viviendo en esta Comunidad Autónoma están afectados en un porcentaje muy inferior a la media (concretamente, el $2,7 \%{ }^{22}$ ). Sin embargo, en el otro extremo, los "recién llegados" superan dicha media con creces, puesto que un 14\% de los inmigrantes que llevan hasta un máximo de 15 meses viviendo en Andalucía ${ }^{23}$ se encuentra residiendo en infraviviendas. En principio, sin considerar de momento el posible impacto de otros factores, parecería que, en la casi totalidad de los casos, la situación residencial se normaliza con el paso de los años.

Por último, la distribución de los casos según el nivel de ingresos, demuestra que los más afectados por la infravivienda son los inmigrantes que declaran tener niveles de ingresos más bajos, o no tener ningún tipo de ingresos personales. De este modo, un $17,9 \%$ de los inmigrantes que declararon no tener ingresos y un $12,8 \%$ de los que ganaron menos de $500 €$ en el mes de referencia (concretamente, febrero de 2003, es decir el mes anterior a la realización del trabajo de campo) viven en situación de infravivienda. A partir de un nivel de ingresos personales superior a los $750 €$, el porcentaje de casos afectados por la infravivienda se reduce mucho (en el intervalo entre 751 y $1000 €$ baja al $2,8 \%$ ), y a partir de $1000 €$, el fenómeno desaparece.

\section{2) El ratio de hacinamiento}

Es innegable que, en toda definición científica, influyen factores de índole histórica y cultural. Dicho matiz histórico y cultural de las definiciones científicas queda a veces irreconocible incluso para los mismos adeptos; sin embargo, es palpable en el ámbito temático que aquí nos concierne, y mayormente lo es a la hora de establecer a partir de qué relación numérica entre habitaciones y número de habitantes resulta "no digna" la situación residencial. Los precedentes que hemos encontrado en la literatura giran generalmente en torno a ratios de entre dos o tres personas por habitación como línea divisoria entre situaciones dignas y no. Entre estos precedentes, nos hemos decantado en principio por una propuesta (Renes, 2002) que considera hacinamiento un ratio inferior a 0,5 habitaciones por persona (o lo que es lo mismo, dos personas por habitación), ya que las definiciones que toman como referencia más de tres personas por habitación suelen referirse al ámbito iberoamericano, donde podrían influir consideraciones culturales en cuanto a la ocupación del espacio distintas de las que rigen actualmente en la sociedad española, relativas al papel de la intimidad personal y familiar para unas condiciones de vivienda digna. Por tanto, vamos a considerar hacinamiento cuando hay más

\footnotetext{
${ }^{22}$ Recordamos que la media se sitúa en un $8,9 \%$.

${ }^{23}$ Nos referimos a los últimos 15 meses antes de la realización del trabajo de campo de la encuesta NEPIA, es decir, el año 2002 y los primeros meses del año 2003.
} 
de dos personas por habitación ${ }^{24}$, reservando a un ratio de más de tres personas por habitación la calificación de "hacinamiento grave".

\section{Extensión y localización del fenómeno}

Si relacionamos el número de personas con el número de habitaciones de que se dispone, nos encontramos un promedio de 1,23 personas por habitación para el conjunto de la población inmigrante de países menos desarrollados afincada en Andalucía, lo cual se traduce en que alrededor del $40 \%$ de los inmigrantes entrevistados dispone de más de una habitación por persona (situación que es particularmente frecuente entre los iberoamericanos, con el $46,4 \%$ ), y casi el $25 \%$ dispone de una habitación por persona. Aproximadamente, en el $6 \%$ de los casos, conviven dos personas por habitación, y en el 9,2\%, más de dos personas por habitación.

Esta última situación, que se corresponde con nuestra definición de hacinamiento, se concentra esencialmente en los municipios de "agricultura intensiva", donde el hacinamiento se extiende a un $14 \%$ de las viviendas o alojamientos

\footnotetext{
${ }^{24}$ El indicador manejado por la Fundación FOESSA (1998: 288) para situaciones de "promiscuidad" (sic), equiparable a nuestra medición de hacinamiento, se refiere a menos de un dormitorio por cada dos personas. En NEPIA, hemos definido como "habitación" de una vivienda los dormitorios y el salón (u otro tipo de habitaciones excepto cocina, baños, trastero, etc), si hubiese, o en su defecto, la unidad habitacional existente, incluyendo las situaciones de infravivienda referidas antes. Para poder calcular el ratio, hemos tenido que transformar la variable referida al número de habitaciones, ya que esta pregunta sólo se hacía a las personas que habían declarado habitar una vivienda completa $(\mathrm{n}=1520)$. Hemos considerado que las personas a las que no correspondía responder a esta pregunta, en general están viviendo en una sola unidad habitacional, ya que por una parte se trata de personas que han declarado vivir en una habitación, y por otra de habitantes de alojamientos precarios, muchos de los que pueden equivaler, con los matices correspondientes, a unidades habitacionales singulares. Como resultado de estas operaciones, el número de casos de la variable "número de habitaciones" es 1784. Por su parte, en la variable referida al número de habitantes, hemos considerado a las personas que viven con sus patrones (que habían quedado recogidas por separado) como si vivieran solas y las respuestas "NC" como valores perdidos, resultando un número de casos igual a 1790. Una vez hecho el cociente entre ambas variables obtenemos 1777 casos. Con el fin de evitar distorsiones en el porcentaje de hacinados, no se van a tener en cuenta los casos en los que se declara convivir con 12 o más personas por habitación (se piense por ejemplo en la posibilidad de que algunos entrevistados alojados en residencias colectivas pueden haberse referido, al indicar este número, a dormitorios amplios que, a pesar de carecer de tabiques de separación, y por tanto implicar una falta de intimidad, no suponen necesariamente una situación de hacinamiento; se piense, por otra parte, también en la posibilidad de que algunos entrevistados se refieran al total de personas alojadas en la pensión u hostal, por ejemplo). La decisión de descartar los casos a partir de la cantidad de 12 personas por habitación no es arbitraria, sino que responde al hecho de que a partir de esta cantidad, se trata sólo de casos de alojamiento precario, lo que hace más probable las posibles distorsiones que hemos señalado. Con esta decisión se descartan 8 casos, siendo el número final de casos válidos $\mathrm{n}=1769$.
} 
ocupados por inmigrantes, mientras que en las zonas turísticas costeras sólo alcanza al $6 \%$.

Para matizar este resultado general, que comprende situaciones de hacinamiento relativo y grave, respectivamente, cabe señalar que el intervalo de entre 2,1 y 3 personas por habitación agrupa a prácticamente la mitad de las personas clasificables como "hacinados" (concretamente, un 4,5\%). Superando las tres personas por habitación, los casos se concentran cada vez más en las zonas de agricultura intensiva, ya que el 9,4\% de los inmigrantes que viven en esta zona está expuesto a hacinamiento grave ${ }^{25}$. En las zonas turísticas costeras, sólo encontramos al $2,1 \%$ de los inmigrantes viviendo en lo que hemos denominado hacinamiento grave.

\section{Hacinamiento: perfil social de los afectados}

Si analizamos la distribución por sexo, vemos que el hacinamiento grave, tal y como lo hemos descrito, afecta en menor medida a las mujeres $(2,6 \%)$ que a los varones $(6,2 \%)$. Asimismo, por año de llegada a Andalucía, se observa un porcentaje de hacinados inferior a la media entre los inmigrantes que llegaron antes de $1999(4,5 \%)$ y un porcentaje bastante mayor entre los recién llegados, dado que un $13,5 \%$ de éstos vive hacinado. Centrándonos únicamente en los hacinados graves (más de 3 personas por habitación), se acentúa el contraste entre los más asentados y los recién llegados. Destacan por el reducido porcentaje de hacinados graves (entorno al 1,6\%) todos los períodos de llegada anteriores al 2000, mientras que entre los recién llegados (hasta 15 meses de permanencia en España), los hacinados graves alcanzan el $8 \%$.

Los ingresos personales en el último mes parecen también guardar relación con la situación de hacinamiento. Las personas sin ingresos son las que tienen un porcentaje más alto de hacinamiento, ya que alcanza al 14,8\% de ellos. En el otro extremo se sitúan las personas con ingresos superiores a los $1000 €$, con sólo un $1 \%$ de hacinados.

Los inmigrantes subsaharianos destacan por el elevado porcentaje (el 16,3\%) que vive en condiciones de hacinamiento. En sentido contrario, se observan porcentajes alrededor del $5 \%$ de afectados en los conjuntos de Asia y de Europa del Este. La misma pauta se reproduce en lo referente al hacinamiento grave, visto que el $10 \%$ de los subsaharianos vive en alojamientos en los que hay más de 3 personas por habitación.

${ }^{25} \mathrm{El} 3,2 \%$ de los inmigrantes asentados en esta zona comparte su habitación con tres personas más, el 2,6\% se encuentrả en situaciones en las que hay 5 personas por habitación y el $1,1 \%$, viviendo con 6 personas por habitación. Los porcentajes de ratios aún superiores no llegan al $1 \%$. 


\section{3) La exclusión espacial}

A la hora de abordar el fenómeno de la exclusión espacial, es importante evitar la confusión con el término "segregación", confusión que sí existe en la literatura disponible. En este artículo, nos centramos en medir la exclusión espacial, debido al hecho de que su relación con la cuestión de la vivienda digna es, con toda evidencia, muy estrecha, pues el alejamiento de los núcleos ${ }^{26}$ habitados puede tener como resultado un escaso o difícil contacto con servicios y equipamientos esenciales que se encuentran ubicados en éstos. Desde luego que, empíricamente, situaciones de exclusión espacial pueden coincidir con lo que denominamos "segregación espacial" (es decir, la concentración de determinadas poblaciones, en función de su procedencia, en zonas circunscritas). Sin embargo, tal coincidencia, si es que existe, es difícil de detectar si no se matiza terminológicamente entre los dos fenómenos. Es más, la equiparación a priori de segregación y de exclusión espaciales impide de antemano analizar la distribución geográficamente dispar de la población inmigrada en relación con sus causas e implicaciones reales.

Para medir la exclusión espacial, hemos elegido tres indicadores relativos al equipamiento del barrio o zona donde se sitúa la vivienda o alojamiento. Para hablar de "exclusión", consideramos necesario que a su alrededor se carezca de acceso a: tiendas y comercios; escuelas o institutos, y transporte público. Existirá un problema de exclusión espacial cuando se den los tres factores juntos, ya que de otro modo la situación no quedaría recogida con suficiente claridad. Pensando en el ámbito rural, una definición que exigiera muchos equipamientos podría tener como resultado la clasificación como "excluidos" de gran parte de la población total.

\section{Extensión y localización del fenómeno}

Si observamos por separado los tres equipamientos cuya ausencia conjunta hemos definido como "exclusión espacial", resulta que la amplia mayoría de los entrevistados manifiesta tener acceso a cada uno de ellos. Concretamente, en orden descendiente, la gran mayoría de los encuestados dispone de acceso a tiendas y comercios $(92,8 \%)$, transporte público $(87,8 \%)$, y escuelas o institutos (78,8\%).

\footnotetext{
${ }^{26}$ Cabe señalar que el Instituto de Estadística de Andalucía define como "núcleo" de población a "un conjunto de al menos diez edificaciones, que están formando calles, plazas y otras vías urbanas. Por excepción, el número de edificaciones podrá ser inferior a 10 , siempre que la población que habita las mismas supere los 50 habitantes. (...)" (http://www.juntadeandalucia.es/institutodeestadistica/ sima/ catalogo/fb05.htm).
} 
RIS

REVISTA INTERNACIONAL DE SOCIOLOCIA

Tabla 1.

Equipamiento de la zona por categoria de vivienda o alojamiento (excepto "habitación"), Andalucia, primavera de 2003

\begin{tabular}{lcc}
\hline & \multicolumn{2}{c}{ Tipo de vivienda } \\
\cline { 2 - 3 } Equipamientos y servicios & Completa & Precaria \\
\hline Guarderías & $61,6 \%$ & $38,5 \%$ \\
Escuelas o institutos & $82,4 \%$ & $53,9 \%$ \\
Transporte público & $89,7 \%$ & $68,6 \%$ \\
Centro de salud & $83,3 \%$ & $59,0 \%$ \\
Zonas verdes & $77,5 \%$ & $47,9 \%$ \\
Tiendas y comercios & $95,1 \%$ & $73,3 \%$ \\
Instalaciones deportivas & $62,4 \%$ & $43,6 \%$ \\
TOTALES & 1508 & 94 \\
\hline
\end{tabular}

Fuente: Encuesta NEPIA

Sin embargo, el grado de equipamiento de la zona residencial es diferente según el tipo de domicilio en el que residen los inmigrantes. En general, como puede observarse en la tabla 1, los barrios o zonas de los inmigrantes que cuentan con una vivienda completa (unifamiliar o compartida), resultan considerablemente más equipados que las zonas de aquellos inmigrantes ubicados en espacios clasificables de entrada como precarios ${ }^{27}$.

Ahora bien, pasando a la suma de las tres ausencias recogidas en nuestra definición de "exclusión", de todos los entrevistados, está en situación de exclusión espacial un 2,6\%. Estas personas se encuentran viviendo principalmente en zonas de agricultura intensiva, donde alcanzan el $7,8 \%$ de todos los inmigrantes asentados.

\section{Perfil social de los afectados}

La exclusión espacial es el fenómeno menos extendido de los tres que nos hemos propuesto medir en este artículo, y es también el que tiene el perfil de afectados menos definido. Sólo el sexo y el conjunto geopolítico de procedencia parecen

${ }^{27}$ Retomamos aquí la clasificación explicada en un apartado anterior. Hemos optado por no reproducir aquí los resultados relativos a una tercera categoría (habitación), al incluir en ésta la variante "casa de patrones", que puede introducir cierta distorsión en cuanto a los equipamientos de la zona. 
estar correlacionados con este fenómeno, pues no se observan diferencias estadísticamente significativas por lo que se refiere al año de llegada o a los ingresos personales. De este modo, parece que la exclusión espacial no es una cuestión que se resuelva con el tiempo, ni tampoco con un aumento en los ingresos.

A tenor de nuestros datos, la exclusión espacial entre las mujeres de procedencia inmigrante es prácticamente residual, afectando sólo al 0,7\%, mientras que entre los varones está mucho más extendida, afectando al $4 \%$ de ellos. En cuanto al conjunto geopolítico de procedencia, un 5\% de los encuestados procedentes del Magreb y Oriente Próximo vive en situación de exclusión espacial, mientras que los inmigrantes asiáticos y los iberoamericanos sufren en mucha menor medida este tipo de situaciones, alcanzando sólo el 0,3\% y el 0,6\%, respectivamente.

\section{4) La acumulación de carencias}

Después de haber definido y descrito por separado los tres fenómenos concretos en los que se puede plasmar la falta de vivienda digna de los inmigrantes asentados en Andalucía, nos parece interesante presentar brevemente algunos datos que revelen en qué medida dichos fenómenos se superponen. La caracterización por perfiles sociales será escueta, bien por el escaso número de afectados por varios de estos fenómenos (número, por tanto, que no permite la elaboración de datos estadísticamente fiables), bien por coincidir el perfil de los afectados por sólo un tipo de carencia, lógicamente, con lo dicho anteriormente.

- Tres tipos de carencias. El porcentaje de inmigrantes alojados en viviendas "triplemente indignas" es realmente bajo, ya que sólo afecta al 0,6\% de los entrevistados, todos ellos asentados en municipios almerienses con economía predominantemente de agricultura intensiva. De este $0,6 \%$ de inmigrantes con tres problemas, el $95 \%$ es de origen magrebí o de Oriente Próximo y el restante $5 \%$ procede del África Subsahariana. Todos los casos en los que observamos la acumulación de los tres tipos de carencias, y por tanto una situación verdaderamente dramática, son varones que han tenido unos ingresos inferiores a $750 €$ en el mes anterior a la encuesta y cuyo año de llegada a Andalucía es posterior al 1999 (el 62\% de ellos lleva un máximo de 15 meses en esta comunidad).

- Dos tipos de carencias. El porcentaje de entrevistados que acumula dos tipos de carencias alcanza al 2,7\%. Los dos problemas que se dan con más frecuencia juntos son los de hacinamiento e infravivienda $(2,1 \%)$, mientras que prácticamente no encontramos casos en los que coincidan el hacinamiento y la exclusión espacial, por un lado, o la infravivienda y la exclusión espacial, por otro. Como no puede ser de otra manera, el perfil de estas personas no difiere en gran medida de lo dicho hasta el momento, a saber: la acumulación de dos problemas de alojamiento es padecida en mayor medida por las personas con ingresos bajos, que viven 
en zonas de agricultura intensiva ${ }^{28}$, y que principalmente provienen del África subsahariana y del Magreb y Oriente Próximo, con más de un 5\% de afectados por dos carencias en cada uno de estos conjuntos geopolíticos. Si cruzamos las dos variables que más impacto parecen tener, observamos que algunas zonas socioeconómicas llevan asociada la precariedad residencial de determinados colectivos de procedencia. Así, en las zonas turísticas costeras sólo encontramos europeos del Este con dos tipos carencias (concretamente, el 2,4\% de ellos). En las grandes ciudades encontramos un porcentaje inferior a la media de inmigrantes subsaharianos, $0,7 \%$, frente al $4,3 \%$ de los magrebíes, mientras que en las zonas de agricultura intensiva, las malas condiciones de alojamiento se concentran en los inmigrantes procedentes del África Subsahariana (15\%) y en los del Magreb y Oriente Próximo (9\%).

- Una carencia. El porcentaje de inmigrantes expuestos a uno de los tres tipos de problemas alcanza casi el $13 \%$ en toda Andalucía, pero como hemos visto examinando una por una las carencias, existe un predominio de las zonas de agricultura intensiva como cuna de los problemas residenciales de los inmigrantes, predominio que se mantiene con independencia del año de llegada de los inmigrantes y de su nivel de ingresos. Sin embargo, es sensible al conjunto geopolítico de procedencia, en el sentido de que carecen de vivienda digna, por padecer uno de los problemas descritos, el $28 \%$ de los inmigrantes de Magreb y Oriente Próximo residentes en las zonas de agricultura intensiva y extensiva, respectivamente. Resulta que, independientemente de dónde residan (e independientemente, en gran medida, también de su año de llegada y nivel de ingresos), los inmigrantes procedentes del Magreb y Oriente Próximo destacan por la mayor frecuencia con que están expuestos a una de las carencias recogidas en nuestra definición de "vivienda no digna" (el 17,8\%), mientras que para los asiáticos $(5,3 \%)$, seguidos por los iberoamericanos $(8,9 \%)$, vale la observación expuesta.

-Vivienda digna. Después de explicar los diferentes problemas de los inmigrantes en relación con la vivienda, parece adecuado finalizar este apartado haciendo referencia a los inmigrantes que no tienen ningún problema de este tipo, y por la tanto residen en una vivienda digna. Es importante resaltar esta cuestión, pues la mayoría de los inmigrantes entrevistados, el $83,2 \%$, reside en una vivienda digna, aunque encontramos zonas en las que la situación es peor que en otras. Las zonas turísticas costeras resaltan por el elevado nivel de inmigrantes, el $91 \%$, que residen en viviendas sin problemas que afecten a su clasificación como "dignas".

${ }^{28} \mathrm{El} \mathrm{4,1 \%} \mathrm{de} \mathrm{las} \mathrm{personas} \mathrm{con} \mathrm{unos} \mathrm{ingresos} \mathrm{inferiores} \mathrm{a} 500 €$ acumula dos carencias, frente al $0,1 \%$ de las personas que cobraron más de $1000 €$. En la zona de agricultura intensiva, un $6,3 \%$ de los entrevistados padece dos tipos de carencias, frente a sólo el $0,3 \%$ en las zonas turísticas costeras. 
Sin embargo, las zonas de agricultura intensiva son las que tienen el porcentaje más bajo de vivienda digna, ya que sólo alcanza el 70,1\%. Encontramos muchas diferencias también entre conjuntos geopolíticos de procedencia, pues mientras que los inmigrantes procedentes de Asia e Iberoamérica se encuentran muy por encima de la media, superando ambos el $90 \%$, entre los inmigrantes procedentes del Magreb y Oriente Próximo y del África Subsahariana, sólo alrededor del 75\% dispone de vivienda digna.

\section{DISCUSIÓN DE LOS RESULTADOS}

La encuesta NEPIA confirma que una minoría relevante de la población inmigrante asentada en Andalucía carece de vivienda digna. En este punto esencial, nuestros resultados coinciden con la literatura anteriormente disponible: en algunas zonas de Andalucía, "miles de trabajadores (pernoctan o viven) en inmuebles que no reúnen los requisitos mínimos (...) de seguridad, salubridad e higiene" (Girón Caro, 2002: 92-3). Por tanto, cabe respaldar al Defensor del Pueblo Andaluz en su insistencia ante las Administraciones Públicas para que dediquen la voluntad política, el esfuerzo y los recursos ${ }^{29}$ necesarios para abordar, junto con los agentes sociales implicados, un problema de tal gravedad.

Sin embargo, la diferencia porcentual entre los resultados de este estudio, por un lado, y lo expuesto en la literatura anteriormente disponible, por otro, es muy llamativa. Mientras que los trabajos pre-existentes postulan que, en las zonas de agricultura intensiva de Andalucía, los fenómenos de infravivienda, hacinamiento y segregación espacial afectan a una amplia mayoría de los inmigrantes procedentes de países menos desarrollados, nuestros datos sugieren un diagnóstico bastante distinto. Según la encuesta NEPIA, la falta de vivienda digna afecta a una proporción relevante, pero en absoluto mayoritaria de la población inmigrante residente en municipios con economía predominantemente de "agricultura inten-

\footnotetext{
${ }^{29}$ Para mejorar la situación residencial de la población inmigrante asentada en Andalucía, después de analizar los desperfectos del Decreto 2/2001 (modificado por el Decreto 291/2003), con el que se había intentado fomentar la construcción de viviendas y alojamientos en alquiler en municipios de alta movilidad laboral, la Consejería de Obras Públicas y Transportes de la Junta de Andalucía apuesta por una diversificación de sus actuaciones. El IV Plan Andaluz de Vivienda y Suelo 2003-2007 (Decreto 149/2003 de 10 de junio de 2003; BOJA núm. 117/2003) incluye una serie de herramientas en este sentido, entre las que señalamos: la promoción de viviendas protegidas de régimen especial en venta (Art. 18-22), la promoción de alojamientos protegidos en alquiler destinados a personas con recursos limitados y necesidades temporales de vivienda (Art. 36-39), el apoyo de bolsas locales de viviendas para alquiler (Art. 52-5) y la transformación de la infravivienda (Art.61-64). Cabe subrayar que se trata de herramientas generales, aplicables a toda la población andaluza en función de su situación social. En relación con el procedimiento de distribución entre los aspirantes (mediante sorteo), los promotores públicos pueden establecer cupos para determinados colectivos sociales especialmente vulnerables.
} 
RIS

REVISTA INTERNACIONAL DE SOCIOLOCIA

№ 38, MAYO-ACOSTO, 2004

SEBASTIAN RINKEN Y ANAÏS HERRÓN

siva", mientras que en toda la población inmigrante asentada en Andalucía, dicha proporción se sitúa por debajo del $20 \%$. Dicho al revés, aproximadamente cuatro de cada cinco inmigrantes procedentes de países menos desarrollados disponen de vivienda o alojamiento digno.

Sin descartar la posibilidad de que la variable tiempo pueda jugar un papel relevante ${ }^{30}$, a continuación aportaremos elementos de una explicación de las diferencias porcentuales observadas, centrándonos en cuestiones metodológicas. Visto que, de todos nuestros resultados, el más sorprendente - pensando en los resultados de estudios anteriores-se refiere a la exclusión espacial, centraremos estas reflexiones metodológicas en dicho fenómeno. Según nuestros datos, lejos de afectar a una mayoría de los inmigrantes asentados en zonas de agricultura intensiva (como venía suponiendo la literatura relativa a la segregación espacial), la exclusión espacial afecta a menos del $8 \%$ de los inmigrantes residentes ên dicha zona socioeconómica. ¿Cómo explicar esta diferencia?

Empezaremos por considerar la posibilidad de que nuestro resultado podría constituir una infraestimación, atribuible al procedimiento de captación de entrevistados aplicado por NEPIA. Recordemos que dicha captación estaba basada en la diversificación de los puntos de contacto con los entrevistados, con cuotas mínimas y máximas relativas a las cuatro categorías en las que se agruparon estos puntos de contacto (laboral, residencial, recreativo y asociativo). Este procedimiento se diseñó con la intención de evitar sesgos de medición debidos al grado de visibilidad de determinados segmentos de la población inmigrada y, en términos generales, dio un resultado muy bueno. Ahora bien, nuestro procedimiento podría haber encontrado cierto límite de representatividad precisamente en las zonas de agricultura intensiva, en la medida en que la lógica inclinación de los entrevistadores a maximizar el rendimiento de su jornada laboral afectara negativamente a la posibilidad de reflejar, en el trabajo de campo, la dispersión territorial de la población objeto. Al no diseñar reglas específicas para extender la captación de los entrevistados al entorno "diseminado" de dichos núcleos, cabe suponer que la gran mayoría de las entrevistas realizadas por los entrevistadores de NEPIA en zonas de agricultura intensiva se efectuaron en alguno de los núcleos de población de los municipios asignados. Como consecuencia, cabe suponer que nuestro procedimiento derivó fundamentalmente en la captación de personas que se encontrasen en dichos núcleos de población, por el motivo que fuera (laboral, residencial, comercial, etc.), quedando al margen aquellas personas cuyas actividades cotidianas se desarrollasen exclusivamente o en mayor medida en el campo (diseminado). Es más, a pesar de contar, en el estudio NEPIA, con mecanismos que evitan la limitación de la captación a los inmigrantes residentes

\footnotetext{
${ }^{30}$ Nuestra recogida de datos se efectuó en la primavera de 2003, mientras que la mayoría de los estudios mencionados en la Introducción de este artículo se hicieron durante el año 2000.
} 
en estos núcleos (al extenderse la captación a vías públicas y otros lugares de agregación), es razonable suponer que la probabilidad de selección de los residentes es mayor que la de personas no residentes en estos núcleos.

A primera vista, algunos de los resultados de NEPIA parecen avalar la hipótesis de que este factor puede incidir mucho en la explicación de la mencionada diferencia. A estos efectos, llaman la atención los resultados relativos a los equipamientos o servicios disponibles en el barrio o zona de residencia. Como apuntábamos en el apartado de resultados, el nivel de equipamiento indicado por nuestros entrevistados es generalmente elevado. Concretamente, para centrarnos en un recurso cuya distribución en el territorio se puede comprobar con relativa facilidad, en el conjunto de la muestra ponderada se observan porcentajes de alrededor del $80 \%$ de acceso a Centros de Salud en el barrio o zona propios. Este porcentaje apenas varía según la zona socioeconómica de asentamiento, alcanzando aproximadamente el $75 \%$ incluso entre los inmigrantes varones residentes en zonas de agricultura intensiva. Visto que, en el amplio territorio del municipio de El Ejido, por ejemplo, el Instituto de Estadística de Andalucía contabiliza sólo tres Centros de Salud, parece imponerse la conclusión de que la encuesta NEPIA infraestima de manera importante la población inmigrante asentada en diseminado.

Sin embargo, sin descartar la posibilidad de que nuestra encuesta pudiera padecer cierta infracaptación de entrevistados viviendo en diseminado, quisiéramos señalar una serie de factores que nos parecen indicar que, si acaso, este sesgo tiene un efecto reducido. Concretamente, nos referimos a los siguientes factores, relativos a la interpretación de términos como el propio "barrio" en el que tener (o carecer de) acceso a determinados equipamientos:

- Para seguir con el ejemplo acerca de la atención sanitaria, consideramos probable que, en las zonas rurales, muchos entrevistados identificaran como "Centro de Salud" no sólo los así denominados centros de atención primaria propiamente dichos, sino también otros recursos como, por ejemplo, los consultorios de atención primaria, de los que en el término municipal de El Ejido existen siete.

- Tampoco excluiríamos la posibilidad de que, para muchos entrevistados residentes en el ámbito rural, la percepción del propio barrio o zona de residencia resulta mucho más amplia de la que contemplaba el manual del entrevistador de la encuesta NEPIA (accesibilidad en 20 minutos a pié) ${ }^{31}$. En este contexto, cabe señalar que, a tenor de los mapas municipales incluidos en el estudio de Castaño Madroñal (2000), un elevado porcentaje de los asentamientos clasificados como "en diseminado" se encuentra a una distancia inferior a tres kilómetros de los núcleos urbanos.

\footnotetext{
${ }^{31}$ Las explicaciones del manual sólo se mencionaban en el caso de que hicieran falta aclaraciones, de modo que, normalmente, la respuesta recogida se refería sin más explicaciones a la pregunta "Ahora le voy a leer una serie de equipamientos servicios. Dígame si en su barrio existen: (...)".
} 
R IS

REVISTA INTERNACIONAL DE SOCIOLOGIA

- Finalmente, cabe señalar que los datos sobre la situación jurídica y laboral de nuestros entrevistados en zonas de agricultura intensiva en absoluto se corresponden con las características del segmento minoritario de la población inmigrante que, a tenor de los estudios existentes con anterioridad al nuestro, se encontraría asentado en los núcleos urbanos. En este sentido, cabe apuntar, por ejemplo, que de nuestros entrevistados en la zona socioeconómica de "agricultura intensiva", un $29 \%$ se encontraba sin empleo en el mes de referencia (Febrero de 2003), mientras que el $49 \%$ de los que sí tenían empleo, no cotizó a la Seguridad Social. Asimismo, cabe señalar que en esta zona de asentamiento, con sólo un $61 \%(54 \%$ para varones y $72 \%$ para mujeres), la proporción de los inmigrantes que están empadronados en su municipio de residencia es sensiblemente más baja que en otras zonas de Andalucía.

En resumen, consideramos que nuestro trabajo presupone un importante avance en cuanto a la sistematicidad y claridad de los procedimientos de medición aplicados y, por tanto, también en cuanto a la validez de los resultados obtenidos. Nuestra explicación de la abultada diferencia frente a los estudios preexistentes se centra, principalmente, en el hecho de que medimos otro fenómeno. Resulta que la exclusión espacial, definida como falta de acceso a determinados servicios o equipamientos básicos, no coincide con el fenómeno de la segregación espacial. Lógicamente, dicha diferencia entre los fenómenos descritos genera importantes diferencias también en cuanto a sus implicaciones de cara a la anhelada integración social de los inmigrantes.

\section{CONCLUSIONES}

Sin restar un ápice a la urgencia con la que afrontar los problemas de infravivienda, hacinamiento y exclusión espacial, nuestros datos invitan a redimensionar el alcance de estos problemas, visto que, de toda la población inmigrante asentada en Andalucía, las graves carencias en cuanto a su situación residencial afectan sólo a una minoría. Escribimos "sólo" una minoría, no por pensar que fuese irrelevante o poco urgente abordar la mejora de su situación residencial, sino en comparación con los estudios anteriormente disponibles. Cabe subrayar, como resultado principal, que la amplia mayoría de nuestros entrevistados se encuentra viviendo en hogares muy parecidos o idénticos a los habitados por la población autóctona. En muchos casos se tratará de viviendas que distan de ser ideales en cuanto a su estado de conservación, equipamiento, tamaño y ubicación. No obstante, afirmaciones iguales o parecidas cabría hacer también de cara a la situación residencial de muchos autóctonos. En resumidas cuentas, no deja de ser llamativo que, frente a la visión alarmista articulada por parte de la sociedad de acogida en relación con la situación residencial de la población inmigrante (visión que se refleja en algunos de los estudios mencionados en nuestra revisión 
bibliográfica), los propios inmigrantes describen mayoritariamente una situación de relativa normalidad.

No descartamos que dicha diferencia de percepciones esté relacionada con la diferencia conceptual a la que nos hemos referido reiteradamente en este artículo, a saber: la diferencia entre la vivienda no digna (entre cuyos elementos figura la falta de acceso a servicios y equipamientos básicos), por un lado, y la agrupación territorial en relación a afinidades geopolíticas, étnicas o culturales, por otro. Constatamos que esta última preocupa enormemente a una parte relevante de la sociedad de acogida, entre otros motivos por entender que se trata fundamentalmente de un proceso involuntario, debido a la discriminación étnica o racial por parte de otros segmentos de la sociedad de acogida. Sin embargo, desde una perspectiva comparada, cabe señalar que la concentración de los inmigrantes en determinados barrios o zonas de residência no suele corresponder únicamente al efecto de discriminaciones relacionadas con su procedencia, sino también a exigencias prácticas y deseos legítimos de los propios inmigrantes, empezando por el papel vital de sus redes familiares y sociales. En este sentido, sin dudar de la existencia de discriminaciones sobre todo en relación con los inmigrantes procedentes del Magreb, consideramos que tal concentración es problemática en si misma fundamentalmente si se produce en barrios o zonas caracterizados por la existencia de viviendas no dignas, sea por la naturaleza o el estado de las mismas, sea por la disponibilidad de servicios y equipamientos básicos. Es éste, precisamente, el fenómeno concreto cuya difusión hemos querido describir.

\section{REFERENCIAS BIBLIOGRÁFICAS}

ABAD MÁRQUEZ, L. (2002), "Trabajadores inmigrantes en las economías avanzadas. La paradoja de la demanda adicional en mercados con exceso de oferta", en F. J. García Castaño y C. Muriel (eds.), La inmigración en España: contextos y alternativas. III Congreso de la Inmigración en España, Vol. II, (Ponencias) Granada, LDEI., pp.459-468.

ARANGO, J. (2000), "Becoming a Country of Immigration at the End of the Twentieth Century: the Case of Spain", en R. King, G. Lazaridis y C. Tsardanidis (Eds.), Eldorado or Fortress? Migration in Southern Europe, Houndmills y Londres, Macmillan Press.

AZURMENDI, M. (2001), Estampas de El Ejido. Un reportaje sobre la integración del inmigrante, Madrid, Taurus.

CACHÓN, L. (2003), Inmigración y segmentación de los mercados de trabajo en España. Sevilla, Fundación CentrA (Documento de trabajo S2003/02; http://fundación-centra-org).

CASTAÑO MADROÑAL, A. (2000), Informe 2000 sobre la inmigración en Almeria, Sevilla, Consejería de Asuntos Sociales, Junta de Andalucía. 
CHECA OLMOS, J. C. (2002). "La residencia de los inmigrantes en Andalucía: entre el cortijo y el gueto", en L. Serra (coord.), Inmigración extranjera en Andalucia. II Seminario sobre la investigación de la inmigración extranjera en Andalucia, Sevilla, Dirección General de Coordinación de Políticas Migratorias, Consejería de Gobernación, Junta de Andalucía, pp. 103-118.

CHECA OLMOS, J. C. y A. ARJONA GARRIDO (2002), "Exclusión residencial de los inmigrantes marroquies en Andalucía", en F. J. García Castaño y C. Muriel (eds.), La inmigración en España: contextos y alternativas. III Congreso de la Inmigración en España, Vol. II (Ponencias), Granada, LDEI., pp. 657-669.

CORTÉS ALCALÁ, L. (1997), Hablando de la exclusión residencial, Madrid, Caritas.

DEFENSOR DEL PUEBLO ANDALUZ (2001), El alojamiento y la vivienda de los trabajadores inmigrantes en el Poniente almeriense y Campo de Nijar, Sevilla, Junta de Andalucía.

FOESSA (1998), Las condiciones de vida de la población pobre en España. Informe general, Madrid, Fundación FOESSA.

GIRÓN CARO, C. (2002), "El alojamiento y la vivienda del colectivo inmigrante en las provincias de Almería y Huelva", en L. Serra (coord.), Inmigración extranjera en Andalucia. II Seminario sobre la investigación de la inmigración extranjera en Andalucia, Sevilla, Dirección General de Coordinación de Políticas Migratorias, Consejería de Gobernación, Junta de Andalucía, pp. 91-101.

GORDO MÁRQUEZ, M. (2002), La inmigración en el paraíso. Integración en la comarca de Doñana, Sevilla, Consejería de Asuntos Sociales, Junta de Andalucía.

GUALDA CABALLERO, E. (2003), Agricultura andaluza y trabajadores extranjeros: del jornalero andaluz al temporero inmigrante, Sevilla, Fundación CentrA (Documento de trabajo A2003/03; http://fundación-centra-org).

HOGGART, K. y C. MENDOZA (1999), "African Immigrant Workers in Spanish Agriculture", Sociologia Ruralis, n 39 (4), pp. 538-562.

IZQUIERDO, A. (1996), La inmigración inesperada. La población extranjera en España, 19911995, Madrid, Trotta.

IZQUIERDO, A. (Dir.) (2003), Inmigración: mercado de trabajo y protección social en España, Madrid, CES.

LEAL, J. (2002), "Segregación social y mercados de vivienda en las grandes ciudades", Revista Española de Sociología, $\mathrm{n}^{\circ}$ 2, pp. 59-76.

MARTÍN DÍAZ, E., A. CASTAÑO MADROÑAL y M. RODRÍGUEZ GARCÍA (1999), Procesos migratorios y relaciones interétnicas en Andalucia: una reflexión sobre el caso del Poniente almeriense desde la antropología social, Sevilla, Consejería de Asuntos Sociales, Junta de Andalucía.

MARTÍNEZ VEIGA, U. (1999), Pobreza, segregación y exclusión espacial. La vivienda de los inmigrantes extranjeros en España, Barcelona, Icaria. 
MAYA JARIEGO, I. (2001), "Sesgos de medida y problemas de muestreo en las encuestas de poblaciones inmigrantes", Metodologia de Encuestas, $\mathrm{n}^{\circ} 3$, pp.197-214.

MTAS (1996), Vivienda e integración social de los inmigrantes, Madrid, Ministerio de Trabajo y Asuntos Sociales.

PÉREZ YRUELA, M., H. SÁEZ MÉNDEZ y M. TRUJILLO CARMONA (2002), Pobreza y exclusión social en Andalucia, Córdoba, IESA-CSIC.

PONCE SOLE, J. (2004), "Solidaridad, cohesión social y derecho público", en T. Montagut (coord.), Diversidad y convivencia en las ciudades, Barcelona, Fundación Pi i Sunyer (en preparación).

PUMARES, P. (2001), "Castaño Madroñal, A.: Informe 2000 sobre la inmigración en Almería", (recensión), Migraciones $\mathrm{n}^{\circ}$ 10, pp.356-360.

RENES, V. (2002), “Lucha conceptual y operativa”, Revista de Fuentes Estadisticas del INE, $\mathrm{n}^{\circ}$ 63, http://www.ine.es/fuentes/Numero63/paginas/14-15.htm.

RINKEN, S. (2003), "Las condiciones de vida de la población inmigrante: retos metodológicos y procedimientos de medición", Metodología de Encuestas Vol. 5, nº 2, pp.155-173.

RINKEN, S., T. DESRUES, L. RODRÍGUEZ MORCILLO-BAENA, E. RODRÍGUEZ ORTIZ y M. PÉREZ YRUELA (2003), "Cuantía y características de la población inmigrante en Andalucía", en J. Carlos Andreo Tudela (coord.), III Seminario sobre la investigación de la inmigración extranjera en Andalucia, Sevilla, Dirección General de Coordinación de Políticas Migratorias, Consejeria de Gobernación, Junta de Andalucía.

SÁNCHEZ HERNÁNDEZ, A. (2002), "Estado de Bienestar, inmigración y vivienda", en C. Clavijo y M. Aguirre (eds.), Politicas Sociales y Estado del Bienestar en España: las migraciones. Informe 2002, Madrid, Hogar del Empleo.

S.O.S. RACISMO (2001), El Ejido: Racismo y explotación laboral, Barcelona, Icaria.

TERRÉN, E. (2003), "La ironía de la solidaridad: cultura, sociedad civil y discursos sobre el conflicto racial de El Ejido", Revista Española de Investigaciones Sociológicas, nº 102, pp. 125-146. 